\title{
Organic homomolecular crystals with the shortest period of about $4 \AA$ : the role of halogen atoms according to the CSD statistics
}

\author{
O. V. Grineva \\ Chemistry Department of Moscow M. V. Lomonosov State University, Moscow, Russia \\ ovg@phys.chem.msu.ru
}

In this work, I have analyzed the distribution of the shortest periods in homomolecular crystals of organic compounds, in which there are aromatic bonds, the influence of halogen atoms on this distribution was revealed, and energy analysis was carried out for several substances belonging to the group of $4 \AA$-structures, according to the method described in [1]. Crystal structures investigated at normal pressure were extracted from the CSD version 5.41 (November 2019) +3 updates using combinations of querries in ConQuest and the pre-defined best room temperature list [2].

As shown in Fig. 1a, at a large bin size, the histogram for reduced cell $a$ value of all crystals under consideration (set I) can be well described by a single normal distribution function. As the bin size decreases, additional maxima begin to appear on the histogram (Fig. $1 \mathrm{~b}$ ), the position of which remains almost constant when the bin size changes from 0.3 to $0.03 \AA$. Similar distributions were plotted for molecules in which there are halogen atoms in any position (set II) and halogen atoms associated with a non-metal atom forming an aromatic bond (set III).
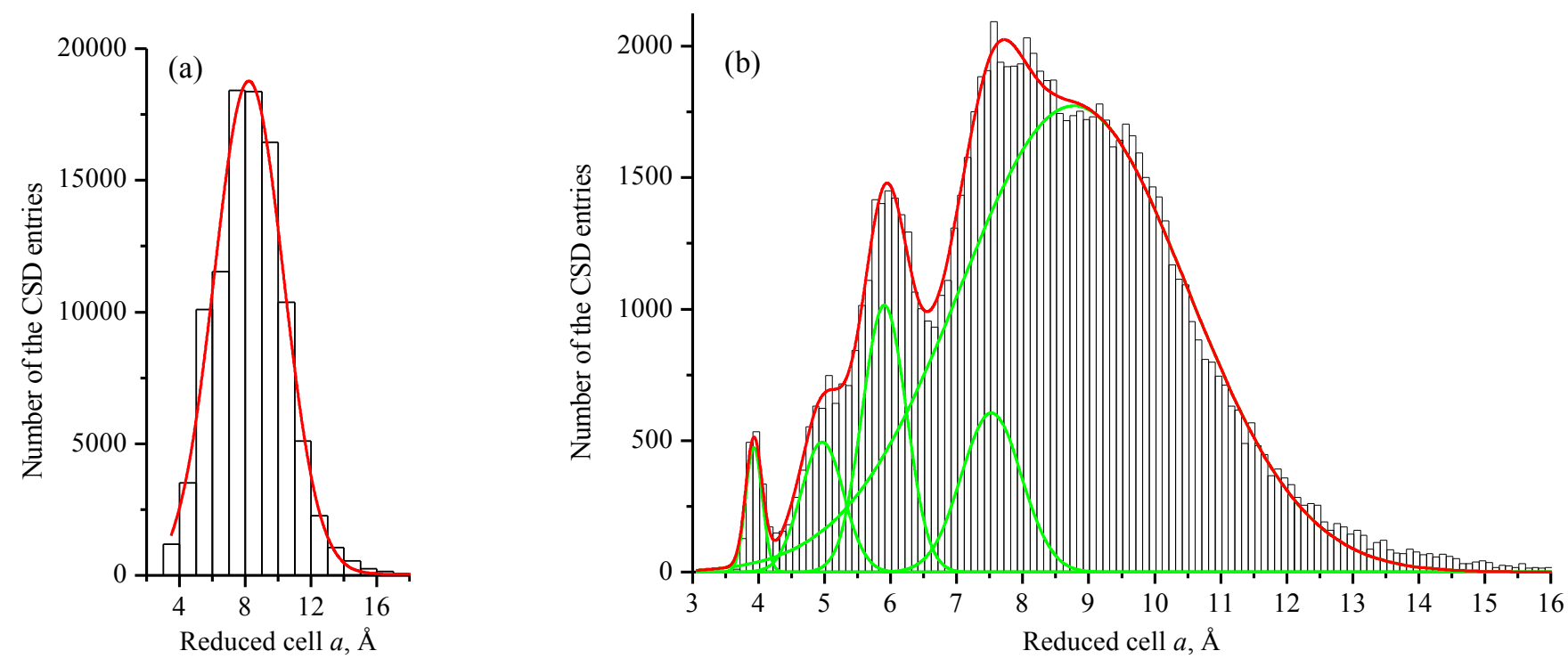

Figure 1. Histograms of the reduced cell $a$ value with the bin size equal to $1 \AA$ (a) and $0.1 \AA$ (b); the red lines show their fitting using one $\left(\mathrm{a}, r^{2}=0.990\right)$ or the sum of five $\left(\mathrm{b}, r^{2}=0.996\right)$ Gaussian functions.

The position of the minimum following the maximum at $3.9 \AA$ for all the above-mentioned sets corresponds to about $4.3 \AA$. It turned out that the parts of substances with the shortest period up to $4.3 \AA$ of the total number of substances in each set are $1.9 \%$ for I, $2.8 \%$ for II, and 3.5\% for III. A reliably determined substance with the shortest period $(3.6021 \AA)$ in all sets is $1,3,4,5,6,8$ hexafluoronaphthalene-2,7-diamine (DAFMUV), in which the contribution of two strongest $\left(E_{1}\right)$ translational molecular contacts to the total packing energy (PE) of the crystal (calculated with Mercury) is 55\% (the energy coordination number, $N_{\mathrm{E}}$, is two). At the other end of all sets is 2-bromo-4-chloro-6-[(2,4-dimethylphenylimino)methyl]phenol (EHUHIZ) with the reduced cell $a$ equal to $4.2995 \AA, N_{\mathrm{E}}=2,2 \cdot E_{1} / \mathrm{PE}=0.48$.

[1] Grineva, O. V. (2017). J. Struct. Chem. 58, 373.

[2] Streek van de, J. (2006). Acta Cryst., B 62, 567.

Keywords: aromatic molecules; infinite stacking; halogen...halogen contacts; packing energy; Cambridge Structural Database The work is a part of research on the theme No. 121031300090-2.

Acta Cryst. (2021), A77, C801 